\title{
Safinamide as Add-On Therapy to Levodopa in Mid- to Late-Stage Parkinson's Disease Fluctuating Patients: Post hoc Analyses of Studies 016 and SETTLE
}

\author{
Carlo Cattaneo ${ }^{\mathrm{a}, *}$, Marco Sardina ${ }^{\mathrm{b}}$ and Ermino Bonizzoni ${ }^{\mathrm{c}}$ \\ ${ }^{a}$ Department of Medical, Zambon SpA, Bresso, Italy \\ ${ }^{\mathrm{b}}$ Department of $R \& D$, Zambon $S p A$, Bresso, Italy \\ c Department of Clinical Science and Community, Section of Medical Statistics and Biometry "GA Maccacaro", \\ University of Milan, Italy
}

Accepted 10 January 2016

\begin{abstract}
.
Background: Studies 016 and SETTLE showed that safinamide was safe and effective as adjunct therapy in patients with advanced Parkinson's disease (PD) and motor fluctuations. The addition of safinamide to a stable dose of levodopa alone or with other antiparkinsonian medications significantly increased ON time with no/non-troublesome dyskinesia, decreased OFF time and improved Parkinson's symptoms.

Objective: To evaluate the clinical effects of safinamide $100 \mathrm{mg} /$ day on motor fluctuations and cardinal Parkinson's symptoms in specific patient subgroups using pooled data from Studies 016 and SETTLE.

Methods: Both studies were double blind, placebo-controlled, randomized, phase 3 trials which enrolled patients with midto late-stage PD experiencing motor fluctuations while receiving optimized and stable doses of levodopa, alone or with other dopaminergic treatments. The present post-hoc analyses assessed the change from baseline in ON time (with no or non-troublesome dyskinesia) and OFF time in subgroups of patients who were receiving only levodopa at baseline, who were classified as "mild fluctuators" (daily OFF time $\leq 4 \mathrm{~h}$ ), and who were receiving concomitant dopaminergic therapy, with or without amantadine, and the effects of safinamide versus placebo on individual cardinal PD symptoms during ON time.

Results: Safinamide significantly increased mean ON time (with no or non-troublesome dyskinesia) and reduced mean OFF time when used as first adjunct therapy in levodopa-treated patients and patients with mild motor fluctuations. Mean daily ON time (with no or non-troublesome dyskinesia) and OFF time were favorably changed, compared with placebo, to similar extents regardless of whether patients were receiving concomitant dopamine agonists, catechol-O-methyltransferase inhibitors and amantadine. Additionally, safinamide improved bradykinesia, rigidity, tremor and gait.

Conclusions: Safinamide was a safe and effective first adjunct therapy in levodopa-treated patients and improved $4 / 5$ cardinal symptoms of PD while providing benefits to mild and non-mild fluctuators and patients receiving other concomitant dopaminergic therapies.
\end{abstract}

Keywords: Parkinson's disease, safinamide, levodopa, MAO-B inhibitor, motor fluctuations, glutamate, adjunct therapy

\footnotetext{
*Correspondence to: Carlo Cattaneo, DBSc CAAAN, Corporate Medical Advisor CNS, Zambon SpA, via Lillo del Duca, 10, 20091 Bresso (Milan), Italy. Tel.: +39 02 66524299;
}

Mobile: +39 347 6174245; Fax: +39 02 66524887; E-mail: carlo.cattaneo@zambongroup.com. 


\section{INTRODUCTION}

Although dopamine replacement therapies such as levodopa and dopamine agonists (DAs) remain the mainstay of the treatment of Parkinson's disease (PD), the long-term use of levodopa is characterized by declining effectiveness over time, and by the development of motor complications. The impairment of dopaminergic and non-dopaminergic pathways are also implicated in the development of motor complications [1].

Safinamide (Xadago ${ }^{\circledR}$, Zambon S.p.A.) is a new drug with a novel multi-modal mechanism of action (dopaminergic and non-dopaminergic) that includes the reversible inhibition of the MAO-B enzyme and the modulation of the glutamate release through the state-dependent blockade of the voltage-gated sodium channels (VGSC) [2-7].

Studies 016 and SETTLE were designed to evaluate the efficacy and safety of safinamide as add-on therapy to a stable dose of levodopa and other PD medications in these patients [8-10].

Considering the large number of patients enrolled and the similarity of trial design between Study 016 and SETTLE, post hoc analyses of the data from the two studies can provide important and clinically relevant information about the effect of adjunctive safinamide treatment on particular PD symptoms and in specific patient subgroups.

This report describes the results of post hoc analyses of pooled data from Study 016 and SETTLE regarding the use of safinamide as adjunctive therapy to determine if: 1) safinamide is effective in improving daily ON time (with no or non-troublesome dyskinesia) and daily OFF time when used as add-on therapy to levodopa in patients with mild motor fluctuations suggestive of early "wearing off"; 2) safinamide improves cardinal motor symptoms of PD in addition to the effect of levodopa during ON time; 3) safinamide added to a combination of levodopa and dopamine agonist or catechol- $O$-methyltransferase (COMT) inhibitor or amantadine therapy is effective in improving daily $\mathrm{ON}$ time (with no or non-troublesome dyskinesia) and daily OFF time.

\section{MATERIALS AND METHODS}

\section{Patients and treatments}

Studies 016 (NCT01187966) and SETTLE (NCT 00627640) were 24-week, phase 3, double blind, placebo-controlled, parallel-group, randomized, multi-center and multinational trials in which patients with mid- to late-stage PD, experiencing motor fluctuations while receiving levodopa (and possibly other dopaminergic treatments), were treated with safinamide or placebo as add-on therapy $[9,11]$. The use of DAs, COMT inhibitors, anticholinergics, and amantadine in addition to levodopa was permitted, provided that they had been taken at a stable dose in the four weeks before screening, while the use of other concomitant MAO-B inhibitors was not allowed. In study 016 there were two doses of safinamide ( 50 and $100 \mathrm{mg} /$ day), while in the SETTLE trial patients started at $50 \mathrm{mg} /$ day and after two weeks increased the dose to $100 \mathrm{mg} /$ day. For this reason in the present post-hoc analysis we have considered only the $100 \mathrm{mg}$ dose. Both protocols and patient materials were approved by Independent Ethics Committees and Health Authorities in all the participating countries. All patients signed an informed consent form and the studies were conducted according to the Declaration of Helsinki.

\section{Outcome measures}

The post hoc analyses reported here compared treatment effects of safinamide $100 \mathrm{mg}$ once daily versus placebo during the double-blind phases of Study 016 and SETTLE. The primary efficacy variable in the studies was the mean change from baseline in daily $\mathrm{ON}$ time with no or non-troublesome dyskinesia as recorded by patients in 18-hour (06.00 to 24.00) diaries. Non-troublesome dyskinesia was defined as dyskinesia that did not interfere with function or cause significant discomfort [12]. Key secondary efficacy endpoints included: changes from baseline to the week 24 endpoint in total daily OFF time, as measured on the diary cards; change from baseline to week 24 in Unified Parkinson's Disease Rating Scale (UPDRS) Part II (activities of daily living) score during ON time; changes from baseline to week 24 in UPDRS Part III (motor function) and Part IV scores (complications of therapy/motor fluctuations) during $\mathrm{ON}$ time.

Study 016 and SETTLE data were pooled to evaluate the primary and the key secondary endpoints in the group of all patients, and in subgroups of patients who were:

- mild fluctuators at baseline (patients with daily OFF time $\leq 4 \mathrm{~h}$ irrespective of concomitant medication),

- patients who were receiving only levodopa at baseline, with at most a combination with a 
peripheral dopamine decarboxylase inhibitor (DDI), benserazide or carbidopa; i.e., no concomitant COMT inhibitors or dopamine agonists, anticholinergics or amantadine,

- patients who were or were not receiving a dopamine agonist (DA) in addition to the baseline stable dose of levodopa (with or without other anti-Parkinson drugs),

- patients who were or were not receiving a COMT inhibitor in addition to the baseline stable dose of levodopa (with or without other anti-Parkinson drugs),

- patients who were or were not receiving amantadine in addition to the baseline stable dose of levodopa (with or without other anti-Parkinson drugs).

The effects of safinamide on the cardinal symptoms and other motor symptoms of Parkinson's disease during ON time were also evaluated: specifically bradykinesia, rigidity, tremor, postural stability, and gait.

\section{Statistical methods}

Comparisons of the mean change from baseline to week 24 for the active-treatment group to placebo were performed using linear effects models with treatment group and study index as fixed dummy effects and baseline value as continuous covariate (ANCOVA analyses). Results are reported as least square means with associated standard errors, twotailed $95 \%$ confidence intervals (CIs) and two-tailed $P$-values. The intention-to-treat (ITT) patient populations were used for all post hoc analyses while the last observation carried forward (LOCF) approach was applied to account for missing data at study termination. No $P$-value adjustments were made for multiplicity generated by secondary and subgroup analyses. SAS software version 9.4 was used for all statistical analyses.

\section{RESULTS}

A total of 1218 patients were enrolled in the two studies, and 1078 patients completed the trials. The pooled dataset for studies 016 and SETTLE comprised 971 patients who received safinamide $100 \mathrm{mg}$ once daily $(n=487)$ or placebo $(n=484)$. Comparisons of the outcomes between safinamide $100 \mathrm{mg}$ and placebo in the pooled database were comparable to the results reported for the individual studies [8-10].

\section{Daily ON time with no/non-troublesome} dyskinesia and daily OFF time

For the primary efficacy variable in both studies, mean changes from baseline (in daily $\mathrm{ON}$ time with no or non-troublesome dyskinesia) were significantly greater than the changes observed with placebo, therefore a highly significant difference with placebo was also obtained when pooling the two studies: $1.42 \mathrm{~h}(95 \% \mathrm{CI}: 1.21,1.64, p<0.0001)$ versus $0.58 \mathrm{~h}$ (95\% CI: $0.37,0.80)$ observed with placebo, mean difference between safinamide and placebo $0.84 \mathrm{~h}$; see Table 1. In the pooled population (safinamide or placebo added to any baseline dopaminergic treatment), safinamide $100 \mathrm{mg}$ reduced the mean total daily OFF time by -1.49 hour (95\% CI: $-1.68,-1.30$ ) from baseline, as compared with a reduction of only -0.63 h (95\% CI: $-0.82,-0.44)$ observed with placebo $(p<0.0001)$, mean difference between safinamide and placebo $-0.86 \mathrm{~h}$ (Table 2).

Least-squares estimates of ON time (with no or non-troublesome dyskinesia) and OFF time changes obtained for the different patient stratifications all provided significant or highly significant differences with placebo.

\section{Safinamide as first adjunct therapy}

A total of 89 patients (10\%) in the pooled dataset comprised the "levodopa only" subgroup (safinamide $n=43$, placebo $n=46$ ), representing patients for whom safinamide was the first antiparkinsonian medication added to levodopa. Adding safinamide $100 \mathrm{mg}$ to levodopa increased the mean $\mathrm{ON}$ time (with no or non-troublesome dyskinesia) by $1.49 \mathrm{~h}(95 \% \mathrm{CI}$ : $0.79,2.19)$, significantly more than did placebo, 0.33 h (95\% CI: $-0.34,1.01, p=0.0206$; mean difference between safinamide and placebo $1.16 \mathrm{~h}$, see Table 1), and significantly reduced the mean OFF time by $-1.59 \mathrm{~h}(95 \% \mathrm{CI}:-2.21,-0.97) \mathrm{com}-$ pared to $-0.23 \mathrm{~h}$ with placebo ( $95 \% \mathrm{CI}:-0.83,0.36$, $p=0.0024$; mean difference between safinamide and placebo $-1.35 \mathrm{~h}$, see Table 2 and Figs. 1A and 2). However, changes significantly different from those observed with placebo were also observed for the larger subgroup of patients for whom safinamide or placebo was not the first add-on to levodopa: mean ON time (with no or non-troublesome dyskinesia) increased by $1.42 \mathrm{~h}(95 \% \mathrm{CI}: 1.20,1.65)$, significantly different from $0.61 \mathrm{~h}$ with placebo $(95 \% \mathrm{CI}$ : $0.38,0.83, p<0.0001$; mean difference between safinamide and placebo $0.82 \mathrm{~h}$, see Table 1 ), whereas OFF time decreased by $-1.49 \mathrm{~h}$ (95\% CI: -1.68 , 
Table 1

Safinamide $100 \mathrm{mg}$ vs. placebo (pooled data): Change from baseline and differences in change from baseline in ON time with no or non-troublesome dyskinesia (Primary endpoint). Least-squares estimates of mean for the pooled ITT population and its stratifications

\begin{tabular}{|c|c|c|c|c|c|c|}
\hline \multirow[t]{2}{*}{ Studies 016 and SETTLE } & \multicolumn{2}{|c|}{$\begin{array}{c}\text { Change with } \\
\text { Safinamide } 100 \mathrm{mg}(\mathrm{h})\end{array}$} & \multicolumn{2}{|c|}{$\begin{array}{l}\text { Change with Placebo } \\
\text { (h) }\end{array}$} & \multirow{2}{*}{$\begin{array}{c}\text { Difference } \\
\text { Safinamide vs. Placebo } \\
\text { mean }[95 \% \mathrm{CI}]\end{array}$} & \multirow[t]{2}{*}{$P$ Value } \\
\hline & $\mathrm{n}$ & mean $[95 \% \mathrm{CI}]$ & $n$ & mean $[95 \% \mathrm{CI}]$ & & \\
\hline Pooled data (ITT pop.) & 487 & $1.42[1.21,1.64]$ & 484 & $0.58[0.37,0.80]$ & $0.84(0.54 \pm 1.15)$ & $<0.0001$ \\
\hline Levodopa (a) only & 43 & $1.49[0.79,2.19]$ & 46 & $0.33[-0.34,1.01]$ & $1.16(0.18 \pm 2.13)$ & 0.0206 \\
\hline Levodopa (a) and other meds. & 444 & $1.42[1.20,1.65]$ & 438 & $0.61[0.38,0.83]$ & $0.82(0.50 \pm 1.14)$ & $<0.0001$ \\
\hline No use of dopamine agonist & 156 & $1.37[1.02,1.72]$ & 149 & $0.49[0.13,0.85]$ & $0.88(0.38 \pm 1.38)$ & 0.0006 \\
\hline Use of dopamine agonist & 331 & $1.47[1.20,1.73]$ & 335 & $0.60[0.34,0.87]$ & $0.86(0.48 \pm 1.24)$ & $<0.0001$ \\
\hline No use of COMT inhibitor & 265 & $1.34[1.05,1.64]$ & 263 & $0.46[0.16,0.75]$ & $0.89(0.47 \pm 1.31)$ & $<0.0001$ \\
\hline Use of COMT inhibitor & 222 & $1.52[1.20,1.83]$ & 221 & $0.74[0.42,1.05]$ & $0.78(0.33 \pm 1.23)$ & 0.0007 \\
\hline No use of amantadine & 373 & $1.37[1.14,1.60]$ & 374 & $0.53[0.29,0.76]$ & $0.84(0.51 \pm 1.17)$ & $<0.0001$ \\
\hline Use of amantadine & 114 & $1.60[1.09,2.11]$ & 110 & $0.77[0.25,1.29]$ & $0.83(0.10 \pm 1.57)$ & 0.0262 \\
\hline Mild Fluctuators (b) & 152 & $0.94[0.59,1.30]$ & 134 & $0.22[-0.15,0.60]$ & $0.72(0.21 \pm 1.23)$ & 0.0062 \\
\hline Non-Mild Fluctuators (b) & 335 & $1.62[1.36,1.89]$ & 350 & $0.74[0.47,1.00]$ & $0.89(0.51 \pm 1.26)$ & $<0.0001$ \\
\hline
\end{tabular}

ITT $=$ intention to treat; $\mathrm{CI}=$ confidence interval; $\mathrm{h}=$ hours; $n=$ number of patients.

Table 2

Safinamide $100 \mathrm{mg}$ vs. placebo (pooled data): Change from baseline and differences in change from baseline in OFF time (Secondary endpoint). Least-squares estimate of means for the pooled ITT population and its stratifications

\begin{tabular}{|c|c|c|c|c|c|c|}
\hline \multirow[t]{2}{*}{ Studies 016 and SETTLE } & \multicolumn{2}{|c|}{$\begin{array}{c}\text { Change with Safinamide } \\
100 \mathrm{mg}(\mathrm{h})\end{array}$} & \multicolumn{2}{|c|}{$\begin{array}{l}\text { Change with Placebo } \\
\text { (h) }\end{array}$} & \multirow{2}{*}{$\begin{array}{c}\text { Difference } \\
\frac{\text { Safinamide vs. Placebo }}{\text { mean }[95 \% \mathrm{CI}]}\end{array}$} & \multirow[t]{2}{*}{$P$ Value } \\
\hline & $\mathrm{n}$ & mean $[95 \% \mathrm{CI}]$ & $n$ & mean $[95 \% \mathrm{CI}]$ & & \\
\hline Poole & 487 & $-1.49[-1.68,-1.30]$ & 48 & $-0.63[-0.82,-0.4$ & $-0.86(-1.13 \pm-0.59)$ & $<0$. \\
\hline Levodopa (a) only & 43 & $1,-0$. & 46 & 6] & & \\
\hline Levodopa (a) and other meds. & 444 & $-1.49[-1.68,-1.29]$ & 438 & $-0.67[-0.87,-0.47]$ & -0.81 & $<0.0001$ \\
\hline No use of dopamine agonist & 156 & $-1.54[-1.83,-1.26]$ & 149 & $-0.54[-0.83,-0.24]$ & $-1.01(-1$ & $<0.0001$ \\
\hline Use of dopamine agonist & 331 & $-1.49[-1.73,-1.25]$ & 335 & $-0.65[-0.90,-0.41]$ & $-0.83(-1.18 \pm-0.49)$ & $<0.0001$ \\
\hline No use of COMT inhibitor & 265 & $-1.45[-1.71,-1.20]$ & 263 & $-0.55[-0.80,-0.29]$ & $-0.90(-1.27 \pm-0.54)$ & $<0.0001$ \\
\hline Use of COMT inhibitor & 222 & $-1.54[-1.82,-1.25]$ & 22 & $-0.74[-1.02,-0.45]$ & $-0.80(-1.20$ & 0.0001 \\
\hline No use of amantadine & 373 & $-1.44[-1.65,-1.23]$ & 374 & $-0.56[-0.77,-0.35]$ & $-0.88(-1.18 \pm-0.58)$ & $<0.0001$ \\
\hline Use of amantadine & 114 & $-1.66[-2.09,-1.23]$ & 110 & $-0.86[-1.30,-0.42]$ & $-0.80(-1.41 \pm-0.19)$ & 0.0110 \\
\hline Mild Fluctuators (b) & 152 & $-0.74[-1.01,-0.47]$ & 134 & $-0.10[-0.39,-0.18]$ & $-0.64(-1.03 \pm-0.24)$ & 0.0018 \\
\hline Non-Mild Fluctuators (b) & 335 & $-1.81[-2.05,-1.56]$ & 350 & $-0.86[-1.10,-0.62]$ & $-0.95(-1.29 \pm-0.60)$ & $<0.0001$ \\
\hline
\end{tabular}

ITT = intention to treat; $\mathrm{CI}=$ confidence interval; $\mathrm{h}=$ hours; $n=$ number of patients.

$-1.29)$, compared to $-0.67 \mathrm{~h}$ with placebo $(95 \% \mathrm{CI}$ : $-0.87,-0.47, p<0.0001$; mean difference between safinamide and placebo $-0.81 \mathrm{~h}$, see Table 2 and Figs. 1A and 2).

\section{Concomitant dopamine agonist (DA) use}

In the pooled ITT population of studies 016 and SETTLE, $68 \%$ of patients were taking stable doses of a DA in addition to levodopa at randomization. For the patients already on DA, adding safinamide $100 \mathrm{mg}$ increased the mean daily $\mathrm{ON}$ time with no/nontroublesome dyskinesia $(1.47 \mathrm{~h}$; $95 \% \mathrm{CI}: 1.20,1.73)$ significantly more than did placebo $(+0.60 \mathrm{~h} ; 95 \%$ CI: $0.34,0.87, p<0.0001$; mean difference between safinamide and placebo $0.86 \mathrm{~h}$ ); a nearly identical mean effect size was observed for the patients not taking baseline DA medications: $1.37 \mathrm{~h}$ with safinamide (95\% CI: $1.02,1.72$ ), as opposed to a lower increment of $0.49 \mathrm{~h}$ with placebo $(95 \% \mathrm{CI}: 0.13,0.85$, $p=0.0006$; mean difference between safinamide and placebo $0.88 \mathrm{~h}$, see Table 1 ).
Concomitantly, adding safinamide reduced the mean daily OFF time as compared to placebo (Table 2 and Figs. 1B and 2) slightly more in the patients who were not taking DA as baseline medication: $-1.54 \mathrm{~h}$ for safinamide $(95 \% \mathrm{CI}:-1.83,-1.26)$, as compared to $-0.54 \mathrm{~h}$ with placebo $(95 \% \mathrm{CI}:-0.83,-0.24$, $p<0.0001$; mean difference between safinamide and placebo $-1.01 \mathrm{~h}$ ) than in patients who were taking DA: -1.49 h (95\% CI: $-1.73,-1.25)$, as compared to $-0.65 \mathrm{~h}$ with placebo $(95 \% \mathrm{CI}$ : $-0.90,-0.41$, $p<0.0001$; mean difference between safinamide and placebo $-0.83 \mathrm{~h}$ ).

\section{Concomitant COMT inhibitor use}

In the pooled ITT population of studies 016 and SETTLE, $45 \%$ of patients were taking stable doses of a COMT inhibitor in addition to levodopa at randomization. In this subgroup of patients (Table 1), safinamide $100 \mathrm{mg}$ increased the mean daily ON time with no/non-troublesome dyskinesia $(1.52 \mathrm{~h} ; 95 \%$ 


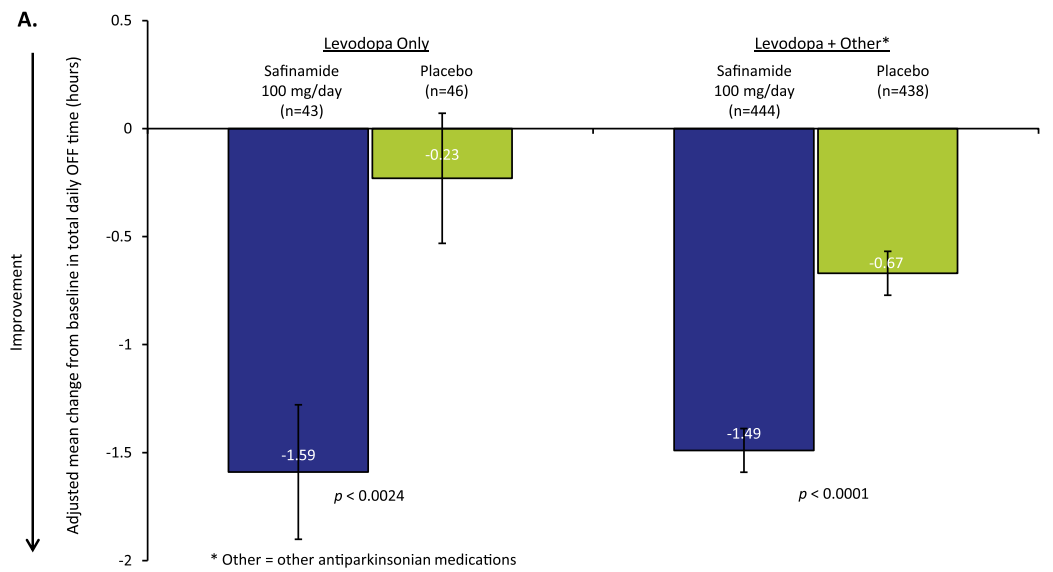

B.
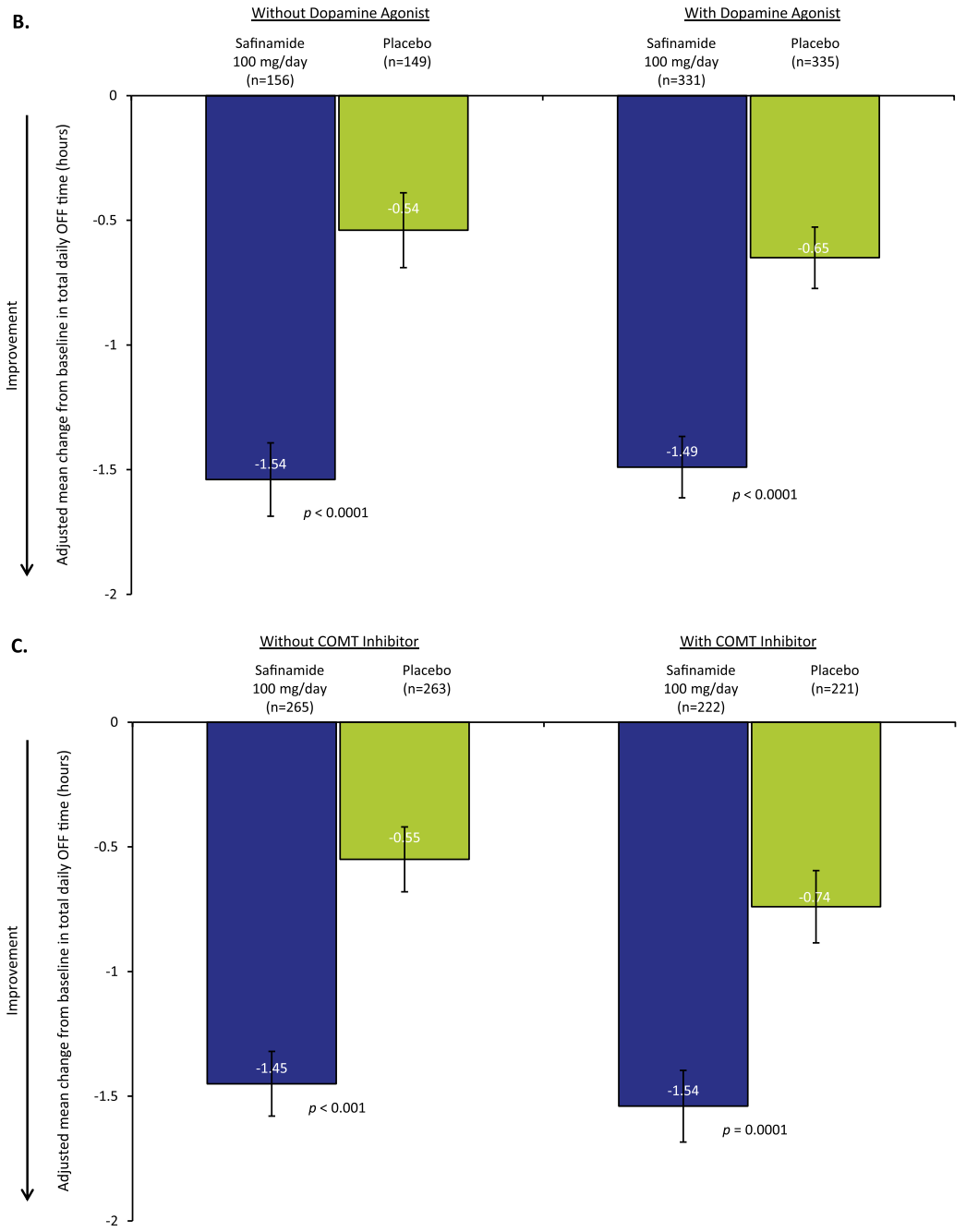

Fig. 1. Pooled data from Studies 016 and SETTLE. Values are least squares mean (LSM) \pm standard error (SE). Adjusted mean change from baseline in total daily OFF time in: A) Patients receiving only levodopa at baseline vs levodopa plus concomitant antiparkinsonian medications, B) Patients receiving and not receiving a dopamine agonist, C) Patients receiving and not receiving concomitant catechol- $O$ methyltransferase (COMT) inhibitor treatment, D) Patients receiving and not receiving concomitant amantadine treatment, E) Patients who were or were not mild fluctuators. 

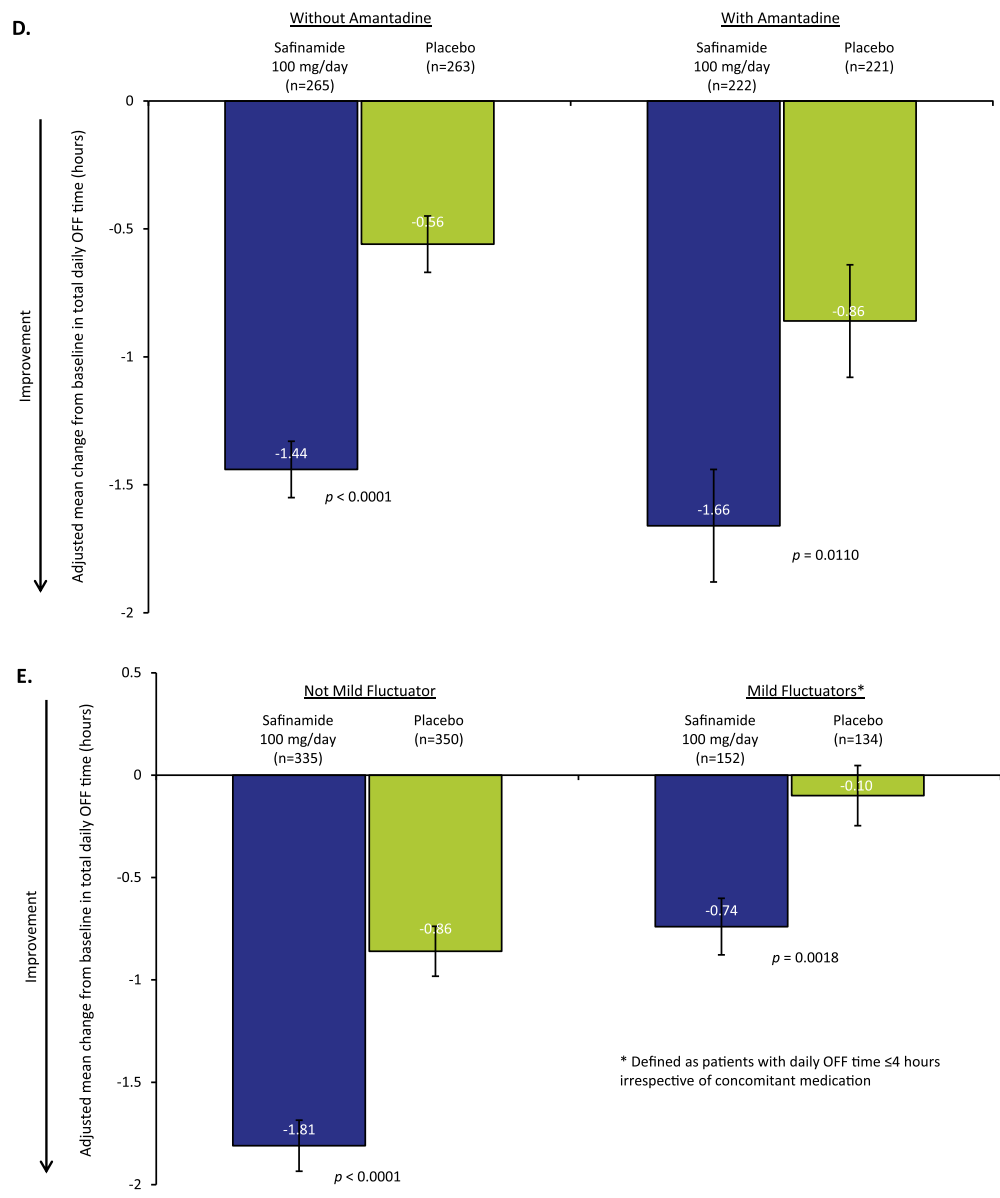

Fig. 1. (Continued)

\section{Subgroup}

Levodopa (a) only Levodope (a) and other meds No use of dopamine agonist Use of dopamine agonist No use of COMT inhibitor Use of COMT inhibitor No use of amantadine Use of amantadine Mild Fluctuators (b) Non-Mild Fluctuators (b)

Mean Difference with $95 \% \mathrm{Cl}$

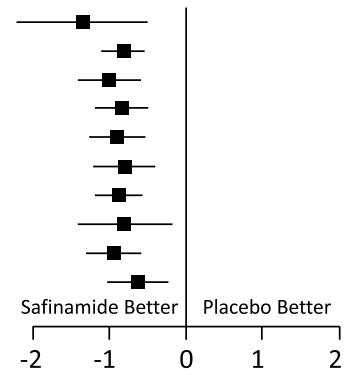

Fig. 2. Pooled data from Studies 016 and SETTLE. Forest plot (OFF time) of the subgroups. Mean difference between safinamide and placebo with 95\% Confidence Interval (CI).

CI: $1.20,1.83)$ significantly more than did placebo $(0.74 \mathrm{~h}$; $95 \%$ CI: $0.42,1.05, p=0.0007$; mean difference between safinamide and placebo $0.78 \mathrm{~h}$ ); the mean effect size was similar to that observed for patients not on COMT inhibitors at baseline, possibly using no or other dopaminergic add-on, which showed comparatively lower mean increments: $1.34 \mathrm{~h}$ with safinamide $(95 \% \mathrm{CI}: 1.05,1.64)$ and $0.46 \mathrm{~h}$ with placebo (95\% CI: 0.16, 0.75, $p<0.0001$; mean difference between safinamide and placebo $0.89 \mathrm{~h}$ ).

The mean daily OFF time (Table 2 and Figs. 1C and 2) decreased with safinamide significantly more than with placebo in the patients already on a COMT inhibitor: $-1.54 \mathrm{~h}(95 \% \mathrm{CI}:-1.82,-1.25)$, as compared to $-0.74 \mathrm{~h}$ with placebo $(95 \% \mathrm{CI}:-1.02,-0.45$, $p<0.0001$; mean difference between safinamide and placebo $-0.80 \mathrm{~h}$ ), but again the mean effect size was not much smaller than that obtained for the patients not on baseline COMT inhibitor therapy: $-1.45 \mathrm{~h}$ with safinamide (95\% CI: $-1.71,-1.20)$ and $-0.55 \mathrm{~h}$ with placebo (95\% CI: $-0.80,-0.29, p<0.0001$; mean difference between safinamide and placebo $-0.90 \mathrm{~h}$ ).

\section{Concomitant amantadine use}

In the pooled ITT population of studies 016 and SETTLE, 23\% of patients were taking stable doses of amantadine in addition to levodopa at randomization. 
In this subgroup of patients (Table 1), safinamide $100 \mathrm{mg}$ increased the mean daily ON time with no/non-troublesome dyskinesia $(1.60 \mathrm{~h}$; $95 \% \mathrm{CI}$ : $1.09,2.11)$ significantly more than did placebo $(0.77 \mathrm{~h}$; $95 \%$ CI: $0.25,1.29, p=0.0262$; mean difference between safinamide and placebo $0.83 \mathrm{~h}$ ); a similar effect size was observed for patients not on amantadine at baseline: $1.37 \mathrm{~h}$ with safinamide $(95 \%$ CI: $1.14,1.60)$ and $0.53 \mathrm{~h}$ with placebo $(95 \% \mathrm{CI}: 0.29$, $0.76, p<0.0001$; mean difference between safinamide and placebo $0.84 \mathrm{~h}$ ).

The mean daily OFF time (Table 2 and Figs. 1D and 2) decreased with safinamide significantly more than with placebo in the patients already on amantadine: $-1.66 \mathrm{~h}$ (95\% CI: $-2.09,-1.23)$, as opposed to $-0.86 \mathrm{~h}$ with placebo (95\% CI: $-1.30,-0.42, p=0.0110$; mean difference between safinamide and placebo $-0.80 \mathrm{~h}$ ), and a similar effect was observed for the patients not taking amantadine on baseline: $-1.44 \mathrm{~h}$ with safinamide (95\% CI: $-1.65,-1.23)$ and $-0.56 \mathrm{~h}$ with placebo (95\% CI: $-0.77,-0.35, p<0.0001$; mean difference between safinamide and placebo $-0.88 \mathrm{~h}$ ).

\section{Mild fluctuators subgroup}

Mild fluctuators (patients experiencing $\leq 4 \mathrm{~h}$ of daily OFF time) were $29 \%$ of the overall pooled ITT population. The addition of safinamide $100 \mathrm{mg}$ to levodopa (plus other possible dopaminergic medications) significantly increased the daily $\mathrm{ON}$ time with no/non-troublesome dyskinesia (Table 1): $0.94 \mathrm{~h}$ (95\% CI: $0.59,1.30)$, as opposed to $0.22 \mathrm{~h}$ with placebo (95\% CI: $-0.15,0.60, p=0.0062$; mean difference between safinamide and placebo $0.72 \mathrm{~h}$ ), but the change was larger, as was the mean effect size in the more serious patients who were experiencing $>4$ h of daily OFF time: $1.62 \mathrm{~h}(95 \% \mathrm{CI}: 1.36,1.89)$, as opposed to $0.74 \mathrm{~h}$ with placebo (95\% CI: $0.47,1.00$, $p<0.0001$; mean difference between safinamide and placebo $0.89 \mathrm{~h}$ ). Consistent with these results, in mild fluctuators the mean daily OFF time decreased significantly relative to placebo: $-0.74 \mathrm{~h}$ with safinamide (95\% CI: $-1.01,-1.47)$, as opposed to $-0.10 \mathrm{~h}$ with placebo (95\% CI: $-0.39,-0.18, p=0.0018$; mean difference between safinamide and placebo $-0.64 \mathrm{~h}$ ), but more substantially in both absolute and relative terms for the non-mild fluctuators $-1.81 \mathrm{~h}$ with safinamide (95\% CI: $-2.05,-1.56)$ and $-0.86 \mathrm{~h}$ with placebo (95\% CI: $-1.10,-0.62, p<0.0001$; mean difference between safinamide and placebo $-0.95 \mathrm{~h}$ ), showing a larger effect size in the more serious patients (Table 2 and Figs. 1E and 2).

\section{Changes in UPDRS scores during ON time}

Changes from baseline in total UPDRS scores and UPDRS subscales during ON time are presented in Table 3.

\section{Activities of daily living}

Safinamide $100 \mathrm{mg}$ significantly improved mean UPDRS Part II (activities of daily living) scores by -1.76 (95\% CI: $-2.09,-1.42$ ) points from baseline, compared with -1.12 (95\% CI: $-1.46,-0.78)$ points with placebo ( $p=0.0097$; mean difference between safinamide and placebo -0.63 points, see Table 3 ).

\section{Motor symptoms}

Safinamide $100 \mathrm{mg}$ significantly improved mean UPDRS Part III (motor symptoms) scores by -5.15 (95\% CI: $-5.87,-4.44)$ points from baseline, compared with -3.25 (95\% CI: $-3.97,-2.53)$ points with placebo ( $p=0.0003$; mean difference between safinamide and placebo -1.90 points, see Table 3 ). With the exception of postural stability $(p=0.7374)$, safinamide $100 \mathrm{mg}$ added to the baseline dopaminergic treatment also significantly improved all the individual cardinal motor symptoms of Parkinson's disease; bradykinesia, rigidity, tremor, and gait (Table 3).

Table 3

Safinamide $100 \mathrm{mg}$ vs placebo (pooled data): Change from baseline and differences in change from baseline in UPDRS scores (Secondary endpoints)

\begin{tabular}{|c|c|c|c|c|}
\hline Studies 016 and SETTLE & $\begin{array}{c}\text { Change with Safinamide } \\
100 \mathrm{mg}(n=482) \\
\text { mean }[95 \% \mathrm{CI}]\end{array}$ & $\begin{array}{c}\text { Change with Placebo } \\
(n=479) \\
\text { mean }[95 \% \mathrm{CI}] \\
\end{array}$ & $\begin{array}{c}\text { Difference } \\
\text { Safinamide vs Placebo } \\
\text { mean }[95 \% \mathrm{CI}]\end{array}$ & $P$ Value \\
\hline UPDRS Part II (ADL) & $-1.76[-2.09,-1.42]$ & $-1.12[-1.46,-0.78]$ & $-0.63(-1.11 \pm-0.15)$ & 0.0097 \\
\hline UPDRS Part III (Motor symptoms) & $-5.15[-5.87,-4.44]$ & $-3.25[-3.97,-2.53]$ & $-1.90(-2.92 \pm-0.88)$ & 0.0003 \\
\hline Bradykinesia & $-2.06[-2.42,-1.7]$ & $-1.39[-1.75,-1.02]$ & $-0.67(-1.18 \pm-0.16)$ & 0.0102 \\
\hline Rigidity & $-1.24[-1.44,-1.04]$ & $-0.74[-0.94,-0.54]$ & $-0.50(-0.79 \pm-0.21)$ & 0.0006 \\
\hline Tremor & $-1.48[-1.7,-1.27]$ & $-0.88[-1.09,-0.66]$ & $-0.61(-0.91 \pm-0.30)$ & 0.0001 \\
\hline Postural stability & $-0.18[-0.25,-0.11]$ & $-0.16[-0.24,-0.09]$ & $-0.02(-0.12 \pm 0.09)$ & 0.7374 \\
\hline Gait & $-0.56[-0.69,-0.44]$ & $-0.34[-0.46,-0.21]$ & $-0.23(-0.40 \pm-0.05)$ & 0.0118 \\
\hline
\end{tabular}

Mean values are least squares estimates [95\% confidence interval (CI)]. ADL, activities of daily living; UPDRS, Unified Parkinson's Disease Rating Scale; $n=$ number of patients. 


\section{Complications of therapy}

There were no statistically significant differences between safinamide and placebo cohorts regarding UPDRS Part IV (complications of therapy) scores; -0.66 (95\% CI: $-0.84,-0.47)$ points from baseline with safinamide $100 \mathrm{mg}$, compared with $-0.44(95 \%$ CI: $0.62,-0.25)$ points with placebo $(p=0.0955)$.

\section{Adverse events}

The incidence of treatment-emergent adverse events (TEAEs), drug-related adverse events, discontinuations due to TEAEs and serious adverse events (SAEs) was similar in safinamide and placebo groups in both studies 016 and SETTLE. The majority of AEs were rated as mild or moderate. Dyskinesia was reported more frequently in the safinamide groups and was generally mild or moderate in severity, transient and did not lead to discontinuations. There were no significant findings for clinical laboratory tests, vital signs, or ophthalmological examination between treatment groups [2, 8-10].

\section{DISCUSSION}

These post hoc analyses of pooled data from these two large phase 3 studies of similar design enables the assessment of the clinical benefits of safinamide across a range of different patient populations. The results were obtained through well-known and standardized scales (UPDRS) scored by the Investigators and by the record of the daily ON and OFF time intervals performed by the patients themselves in their home-diaries.

Stratifications according to the administration of other baseline medications (in addition to a stable dose of levodopa) were not bound to provide a clear trend, since concomitant multiple adjunctive treatments at baseline were admitted in addition to levodopa; in fact, only few patients $(10 \%)$ in the pooled studies ITT population had no additional baseline treatments, i.e., were receiving levodopa as their sole dopaminergic treatment on entering the studies; the majority of patients (90\%) had indeed one or more concomitant baseline treatments, and thus patient subgroups by single adjunctive treatment partly overlapped.

Safinamide demonstrated similar potential to bring about an increase in ON time (with no or nontroublesome dyskinesia) and a concomitant decrease in OFF time relative to the pertinent placebo populations, irrespective of whether or not other medications were already added to the baseline levodopa dose (thus suggesting that safinamide could be an appropriate choice as first adjunct therapy to levodopa in patients with Parkinson's disease experiencing motor fluctuations), and also disregarding the therapeutic class of the possible prior adjunctive therapy (i.e., safinamide being used on top of prior DA or prior COMT inhibitor or prior amantadine therapy).

In both studies 016 and SETTLE, safinamide $100 \mathrm{mg}$ significantly improved overall UPDRS scores for activities of daily living and motor function, compared with placebo, with no deterioration in the UPDRS IV (complication of therapy) [8-10]. This result is important because the patients were receiving a stable, optimized dopaminergic therapy, so further improvements in the UPDRS scores were unexpected.

Safinamide $100 \mathrm{mg}$ significantly improved the UPDRS scores for bradykinesia, rigidity, tremor, and gait, compared with placebo. It is acknowledged that bradykinesia and rigidity are most responsive to levodopa, which has limited effect on postural stability and gait, while the effect of levodopa on tremor is more variable [13]. Although the pooled data did not show an improvement in postural stability with the addition of safinamide, the improvements in tremor and gait are notable and, at least in the case of tremor, likely to be clinically relevant. Importantly, these benefits were not accompanied by a worsening in the activities of daily living.

\section{CONCLUSIONS}

Patients entering studies 016 and SETTLE were experiencing motor fluctuations despite concomitant dopaminergic therapy, reflecting the progressive reduction in the effectiveness of the treatments as Parkinson's disease progresses.

The findings of this post hoc analysis suggest that safinamide can be considered either as a first adjunct medication in Parkinson's disease patients who are not sufficiently controlled on levodopa, and as an add-on in patients already taking levodopa and other concomitant dopaminergic medications (with or without amantadine). The benefits of safinamide were also reflected in improvements in the cardinal symptoms of Parkinson's disease.

\section{CONFLICT OF INTEREST}

Carlo Cattaneo and Marco Sardina are Zambon SpA employees. Erminio Bonizzoni is a statistical consultant of Zambon SpA. 


\section{ACKNOWLEDGMENTS INCLUDING SOURCES OF SUPPORT}

The authors thank Roberto La Ferla for the editorial assistance and Ray Hill, an independent medical writer, who provided medical writing support and journal styling on behalf of Health Publishing \& Services Srl. The authors have no other acknowledgments to disclose.

\section{REFERENCES}

[1] Fox SH (2013) Non-dopaminergic treatments for motor control in Parkinson's disease. Drugs, 73, 1405-1415.

[2] Kulisevsky J (2014) Emerging role of safinamide in Parkinson's disease therapy. Eur Neurol Rev, 9, 1-5.

[3] Caccia C, Maj R, Calabresi M, Maestroni S, Faravelli L, Curatolo L, Salvati P, \& Fariello RG (2006) Safinamide: From molecular targets to a new anti-Parkinson drug. Neurology, 67, S18-S23.

[4] Caccia C, Salvati P, Rossetti S, \& Anand R (2007) Safinamide: Beyond MAO-B inhibition. Parkinsonism Relat Disord, 13, S99.

[5] Chazot PL (2007) Safinamide for the treatment of Parkinson's disease, epilepsy and restless legs syndrome. Curr Opin Investig Drugs, 8, 570-579.

[6] Pevarello P, Bonsignori A, Dostert P, Heidempergher F, Pinciroli V, Colombo M, McArthur RA, Salvati P, Post C, Fariello RG, \& Varasi M (1998) Synthesis and anticonvulsant activity of a new class of 2-[(arylalky) amino]alkanamide derivatives. J Med Chem, 41, 579-590.
[7] Stocchi F, Vacca L, Grassini P, De Pandis MF, Battaglia G, Cattaneo C, \& Fariello RG (2006) Symptom relief in Parkinson disease by safinamide: Biochemical and clinical evidence of efficacy beyond MAO-B inhibition. Neurology, 67, S24-S29.

[8] Anand R, Schapira A, Giuliani R, \& Lucini V (2013) Safinamide is associated with clinically important improvement in motor symptoms in fluctuating PD patients as add-on to levodopa (SETTLE) [Poster]. Mov Disord, 28, S151-S152.

[9] Borgohain R, Szasz J, Stanzione P, Meshram C, Bhatt M, Chirilineau D, Stocchi F, Lucini V, Giuliani R, Forrest E, Rice P, \& Anand R (2014) Randomized trial of safinamide add-on to levodopa in Parkinson's disease with motor fluctuations. Mov Disord, 29, 229-237.

[10] Schapira A, Fox S, Hauser R, Jankovic J, Jost W, Kulisevsky J, Pahwa R, Poewe W, Lucini V, \& Anand R (2013) Safinamide significantly improves responder rates in fluctuating Parkinson's disease (PD) patients as add-on to levodopa (SETTLE) [Poster]. Mov Disord, 28, S152.

[11] Schapira AH, Fox S, Hauser R, Jankovic J, Kulisevsky J, Pahwa R, Poewe W, von Raison F, Kenney C, \& Musch B (2010) SETTLE study design: A 24-week, double-blind, placebo-controlled study of the efficacy and safety of safinamide as add-on therapy to levodopa in patients with Parkinson's disease. Mov Disord, 25, S308.

[12] Hauser RA, Friedlander J, Zesiewicz TA, Adler CH, Seeberger LC, O’Brien CF, Molho ES, \& Factor SA (2000) A home diary to assess functional status in patients with Parkinson's disease with motor fluctuations and dyskinesia. Clin Neuropharmacol, 23, 75-81.

[13] Sethi K (2008) Levodopa unresponsive symptoms in Parkinson disease. Mov Disord, 23(Suppl 3), S521-S533. 